\title{
Punção Aspirativa por Aguulha Fina: Desempenho no Diaǵnóstico Diferencial de Nódulos Mamários Palpáveis
}

Fine Needle Aspiration Biopsy: Performance in the Differential Diagnosis of Palpable Breast Masses

Orlando José de Almeida, Marcelo Alvarenga, José Guilherme Cecatti, Jessé de Paula Neves Jorge, Júlia Kawamura Tambascia

\section{RESUMO}

Objetivo: avaliar, de forma prospectiva, o desempenho da punção aspirativa por agulha fina (PAAF) no diagnóstico diferencial de nódulos mamários palpáveis.

Método: avaliaram-se a sensibilidade, a especificidade, os valores preditivos e a acurácia deste teste em 102 mulheres com idade superior a 30 anos, com nódulos mamários palpáveis, atendidas na Universidade Estadual de Campinas. As punções foram realizadas por um único examinador.

Resultados: o procedimento teve sensibilidade de 97\%, especificidade de 87\%, valor preditivo positivo de $94 \%$ e negativo de 93\%. A taxa de material insuficiente ou insatisfatório foi de $16 \%$ na primeira punção, diminuindo para $2 \%$ com uma nova PAAF.

Conclusões: Este teste mostrou-se altamente sensível e específico no diagnóstico diferencial de nódulos mamários palpáveis, reafirmando-se a sua grande importância na abordagem clínica de nódulos palpáveis.

PALAVRAS-CHAVE: Punção aspirativa por agulha fina. Câncer da mama.

\section{Introdução}

O aumento da incidência do carcinoma de mama nas últimas décadas, com todas suas implicações em termos de morbidade e mortalidade para as mulheres, bem como os gastos decorrentes de seu tratamento e complicações, tem demandado esforços no sentido de aprimorar o arsenal

Departamento de Tocoginecologia - Faculdade de Ciências Médicas - Universidade Estadual de Campinas Correspondência:

Orlando José de Almeida

R. Barão Geraldo de Resende, 282/74

13020-440 - Campinas - SP

Fax: 019-2514586 propedêutico para o diagnóstico precoce dessa condição. O nódulo mamário palpável constitui a principal situação clínica a ser investigada para a diferenciação entre processos patológicos benignos e malignos. Para isso, além do exame clínico e mamografia, a punção aspirativa por agulha fina (PAAF) tem sido utilizada isolada ou conjuntamente, sendo, portanto, necessária a avaliação de seu desempenho.

A PAAF foi proposta como teste diagnóstico, pela primeira vez, em 1930. Martin e Ellis ${ }^{13}$ relataram o uso desse método na abordagem de 65 casos de tumores de diversos órgãos, descrevendo seis casos de punções de mama. Alguns anos mais tarde foram publicados estudos 
em que se avaliaram os riscos relacionados ao exame e as suas principais vantagens e desvantagens, mostrando-se como um bom método diagnóstico ${ }^{2,17,18}$. A partir dessa época, iniciaramse as avaliações com estudos do tipo validação de testes diagnósticos, principalmente em países da Europa $^{21,22}$. Posteriormente, retomaram-se estudos norte-americanos com o uso deste teste, baseandose na excelente experiência obtida nos países escandinavos $^{6}$.

Em recente publicação elaborada pelo Instituto Nacional de Câncer dos Estados Unidos da América, foram reunidas recomendações para o uso da PAAF em lesões mamárias. A importância desse método foi reconfirmada, desde que realizado por profissional com treinamento adequado. Uma taxa de lâminas com material insuficiente inferior a $20 \%$, taxa de falsos positivos inferior a $1 \%$, além de avaliação regular da taxa de resultados falsonegativos fazem parte de critérios de qualidade fundamentais para utilização do método ${ }^{4}$.

A PAAF, que está sendo cada vez mais usada na prática clínica, fornece material para exame da morfologia celular de determinada lesão. Se analisado por citopatologista experiente, é possivel o diagnóstico citológico de uma série de lesões benignas e malignas, baseado nas alterações de celularidade, coesão celular, características do núcleo e da distribuição da cromatina ${ }^{1}$. Desde que realizada com técnica adequada, possui alto índice de concordância com o diagnóstico histológico, podendo ser repetida, se necessário, além de ser um procedimento ambulatorial, com mínimo gasto material.

O uso desse método tem-se ampliado na abordagem de pacientes com nódulos mamários palpáveis, principal forma de apresentação das neoplasias mamárias em nosso país. Entre os métodos usados para o diagnóstico diferencial de lesões mamárias palpáveis, realmente a PAAF parece ser o exame isolado com o melhor desempenho. Realizou-se um estudo prospectivo para a análise do desempenho desse método no diagnóstico de nódulos mamários palpáveis, com avaliação de vários aspectos relacionados ao seu diagnóstico.

\section{Pacientes e Métodos}

O presente estudo foi prospectivo, do tipo validação de testes diagnósticos, com cálculo da sensibilidade, especificidade, valores preditivos positivo e negativo e acurácia da punção aspirativa por agulha fina no diagnóstico diferencial de nódulos mamários palpáveis. O padrão-ouro foi o exame anatomopatológico de todas as lesões incluídas no estudo.

O tamanho calculado da amostra foi de 102 mulheres, considerando-se uma proporção de verdadeiros positivos para malignidade de $90 \%$ e diferença desejável entre a proporção amostral e populacional absoluta de $6 \%$, com erro alfa de $5 \%$.

Foram incluídas mulheres com 30 anos de idade ou mais, com nódulos palpáveis. Excluíramse as pacientes com neoplasia mamária maligna prévia homolateral, e também aquelas cujo nódulo diagnosticado era cístico à punção. Todas as mulheres foram atendidas nos ambulatórios do Centro de Atenção Integral à Saúde da Mulher da Universidade Estadual de Campinas, no período de janeiro de 1996 a julho de 1997.

As PAAF foram realizadas feitas todas por um único examinador, usando-se seringas descartáveis de $20 \mathrm{ml}$, empunhadura metálica, agulhas descartáveis 21 G 1 de 25x8. Após a preensão do nódulo, procedeu-se à punção e aspiração do mesmo com movimentos de ir e vir no interior da massa, até à visualização do material. Prepararam-se, em média, quatro lâminas por nódulo, fixadas em álcool a 95\% mais carbowax a $5 \%$, e depois preservadas em álcool-éter. Foram enviadas ao Laboratório de Citologia do serviço, onde foram coradas com hematoxilina, EA 36 e orange, posteriormente analisadas por médico citologista. Nos casos com materal insuficiente ou insatisfatório para diagnóstico, foi realizada uma segunda punção.

Os diagnósticos citológicos foram fornecidos baseando-se nas alterações de celularidade, coesão celular, características do núcleo (tamanho e forma) e na distribuição da cromatina. Os resultados do exame citológico foram divididos em benignos, suspeitos, malignos e insuficientes/insatisfatórios ${ }^{1}$. $\mathrm{Na}$ análise da acurácia da PAAF, os resultados suspeitos e malignos foram agrupados numa mesma categoria, e aqueles com material insuficiente ou insatisfatório avaliados à parte.

O exame anatomopatológico dos nódulos foi realizado após biópsia excisional na maioria dos casos, ocorrendo somente quatro casos em que se baseou no material de punção de agulha grossa. Os resultados desse exame foram divididos em benignos (incluídas lesões não-neoplásicas e neoplásicas) ou malignos (compativeis com tumores malignos epiteliais, mistos epiteliais / mesenquimais ou de origem diversa).

Para avaliação das médias dos diâmetros dos nódulos foi feita a mensuração, medida em centímetros, da imagem mamográfica. Optou-se por essa medida, apesar de esse exame não fazer parte do atual estudo, por não ser factivel a medida 
precisa de todas as lesões no exame histológico (alguns carcinomas foram submetidos a tratamento quimioterápico prévio à cirurgia).

Para o cálculo da significância estatística das diferenças encontradas na análise da idade e diâmetro médio dos nódulos nos dois grupos (doença maligna ou benigna), usou-se o teste t de Student. Utilizou-se o pacote estatístico Epi.Info versão 6.02 para os diversos procedimentos de análise. $O$ projeto do estudo foi previamente aprovado pela Comissão de Pesquisa e Comitê de Ética da Instituição.

\section{Resultados}

Do total de 102 mulheres, 69\% apresentou doença maligna e $31 \%$ doença benigna. A média de idade foi de 53,4 anos, sendo significativamente superior no grupo com doença maligna em relação ao outro grupo $(p=0,006)$. Analisou-se também o diâmetro dos nódulos mamários, medido nas mamografias. A média geral foi de $2,56 \mathrm{~cm}$, semelhante nos dois grupos $(2,72 \mathrm{~cm}$ nas benignas e 2,49 nas malignas, $\mathrm{p}=0,29$ ).

A PAAF teve alta sensibilidade (97\%), mostrando que apenas dois dos 69 tumores malignos não foram diagnosticados pelo teste (um caso de doença maligna não foi incluído neste grupo por ter resultado insuficiente). A sua especificidade foi de $87 \%$, com valor preditivo positivo de $94 \%$ e valor preditivo negativo de 93\% (Tabela 1).

Tabela 1 - Desempenho do resultado da PAAF para o diagnóstico de malignidade dos nódulos mamários.

\begin{tabular}{lccr}
\multicolumn{4}{c}{ Exame histológico } \\
\hline PAAF & Maligno & Benigno & Total \\
\hline Maligno & 67 & 4 & 71 \\
Benigno & 2 & 27 & 29 \\
Total & 69 & 31 & 100 \\
\hline
\end{tabular}

Sensibilidade $=97 \% . \quad$ Especificidade $=87 \%$.

Valor preditivo positivo $=94 \%$. Valor preditivo negativo $=93 \%$.

Entre os resultados das punções, 23\% do total tiveram o diagnóstico de suspeito para células neoplásicas, sendo analisados juntamente aos positivos. Os suspeitos representaram 34\% do total do grupo considerado maligno. Entre esses casos, a maior parte foi de doença maligna (83\%), com 4 casos de lesões benignas ao exame histológico. A Tabela 2 mostra a distribuição dos diagnósticos anatomopatológicos nesse grupo.
Tabela 2 - Distribuição dos diagnósticos histológicos de nódulos mamários no grupo de casos com resultado citológico suspeito.

\begin{tabular}{lc} 
EXAME HISTOLÓGICO & $\mathbf{n}^{\mathbf{0}}(\boldsymbol{\%})$ \\
\hline $\begin{array}{l}\text { Lesões malignas } \\
\text { carcinoma ductal invasivo } \\
\text { outros carcinomas invasivos }\end{array}$ & $17(17)$ \\
Lesões benignas & $3(12)$ \\
$\quad$ esteatonecrose & \\
fibroadenoma \\
fibrose & $2(8)$ \\
TOTAL & $1(4)$ \\
\end{tabular}

Em 15 casos realizou-se uma segunda punção, pois os resultados da primeira mostraram material insuficiente ou insatisfatório para o diagnóstico citológico. Desses casos, a maior parte foi de lesões benignas, porém o carcinoma ductal invasivo foi a lesão isolada que mais originou essa resposta à punção (Tabela 3).

Tabela 3 - Distribuição dos diagnósticos histológicos de nódulos mamários no grupo de punções com resultados insuficientes / insatisfatório após a primeira punção.

\begin{tabular}{lc} 
EXAME HISTOLÓGICO & $\mathbf{n}^{\mathbf{0}(\%)}$ \\
\hline $\begin{array}{l}\text { Lesões benignas } \\
\text { fibrose } \\
\text { hiperplasia ductal típica } \\
\text { fibroadenoma }\end{array}$ & $2(12)$ \\
$\quad$ esteatonecrose & $1(6)$ \\
$\quad$ ectasia ductal & $2(12)$ \\
$\quad$ tumor filodes benigno & $1(6)$ \\
Lesões malignas & $1(6)$ \\
$\quad$ carcinoma ductal invasivo \\
carcinoma papilífero intracístico & $1(6)$ \\
TOTAL & $6(40)$ \\
\hline
\end{tabular}

Após a segunda punção, apenas 2 casos mantiveram-se como material insuficiente ( 1 caso de carcinoma papilífero intracístico com áreas de carcinoma in situ e 1 caso de fibroadenoma). Na punção do caso com carcinoma intracístico não houve saída de material líquido, sendo por esse motivo incluído no estudo. A taxa de resultados falso-negativos foi de $7 \%$, sem falso-positivos, se incluídos somente os resultados positivos para doença maligna (sem os exames suspeitos).

As Tabelas 4 e 5 mostram a distribuição, de forma detalhada, dos diagnósticos histológicos de todas as lesões avaliadas, tanto as benignas quanto as malignas. 
Tabela 4 - Distribuição dos diagnósticos histológicos nos nódulos mamários com doença benigna.

\begin{tabular}{lc} 
EXAME HISTOLÓGICO & $\mathbf{n}^{\mathbf{0}}(\boldsymbol{\%})$ \\
\hline Fibroadenoma & $14(44)$ \\
Fibrose estromal & $4(12)$ \\
Hiperplasia ductal típica & $3(9)$ \\
Esteatonecrose & $3(9)$ \\
Ectasia ductal & $3(9)$ \\
Lipoma & $2(6)$ \\
Adenose esclerosante & $1(3)$ \\
Tumor filodes benigno & $1(3)$ \\
Cisticercose & $1(3)$ \\
TOTAL & 32
\end{tabular}

Tabela 5 - Distribuição dos diagnósticos histológicos nos nódulos mamários no grupo com doença maligna.

\begin{tabular}{lc} 
EXAME HISTOLÓGICO & $\mathbf{n}^{\mathbf{0}}(\boldsymbol{\%})$ \\
\hline Carcinoma ductal invasivo & $63(90)$ \\
Carcinoma lobular invasivo & $1(1)$ \\
Carcinoma papilífero invasivo & $1(1)$ \\
Carcinoma tubular invasivo & $1(1)$ \\
Carcinoma misto ductal/papilífero & $1(1)$ \\
carcinoma papilífero intracístico & $1(1)$ \\
Doença de Paget do mamilo + Ca ductal inv. & $1(1)$ \\
Linfoma não-Hodgkin metastático & $1(1)$ \\
TOTAL & 70
\end{tabular}

\section{Discussão}

Este estudo confirmou, de maneira prospectiva, as altas sensibilidade e especificidade da PAAF no diagnóstico de nódulos mamários palpáveis, o que concorda com outros estudos $^{3,5,11,14,15,19,20}$. A taxa de exames com material insuficiente ou insatisfatório para o diagnóstico, se considerada uma segunda punção, foi inferior a vários outros autores ${ }^{9,10}$, o que pode ter ocorrido pela homogeneidade na realização das punções, feitas por um só examinador, e pela experiência dos citopatologistas, fundamental na precisa avaliação das lâminas.

O percentual de casos com resultados suspeitos foi superior a de outros estudos ${ }^{8,20}$. Esse grupo foi incluído entre os resultados positivos para malignidade e, como era de se esperar, uma parte desses correspondeu a doenças benignas. Na prática, torna-se necessário incluir os suspeitos nesse grupo, pois a conduta deve ser mais agressiva em tais casos.

Analisadas somente as punções com resultados positivos para doença maligna, a taxa de resultados falso-positivos foi nula. A taxa de falso-negativos foi semelhante a de outros estudos ${ }^{4,23}$.

Esse método diagnóstico tem sido cada vez mais utilizado na prática clínica, pois seu custo é reduzido e o procedimento é ambulatorial ${ }^{12}$. Complicações imediatas ao uso do método, como sangramento ou dor, são muito pouco freqüentes quando se usa técnica adequada ${ }^{10,17}$. A possibilidade de penetração de células neoplásicas no trajeto da agulha da punção de nódulos malignos da mama é mínima e parece não influenciar o tratamento e prognóstico da doença, aparecendo esporadicamente na literatura ${ }^{7}$.

A utilização da punção de agulha fina deve ser estimulada, pois os resultados obtidos com o uso de técnica adequada podem ser muito importantes para orientação terapêutica dos casos. Em nosso meio, onde os recursos para a utilização de exames diagnósticos é reduzido, a utilização de métodos de baixa complexidade e custo reduzido devem ser estimulados.

Convém recordar que a situação ideal para o diagnóstico de um nódulo mamário palpável é o uso combinado de métodos, na forma de diagnóstico tríplice (exame clínico, mamografia e PAAF). A punção aspirativa, dentro desse contexto, é o exame com maior valor isolado, devendo sempre orientar a conduta terapêutica ${ }^{16}$.

\section{SUMMARY}

Purpose: to evaluate, in a prospective way, the performance of the fine needle aspiration biopsy in the differential diagnosis of palpable breast masses.

Method: the sensitivity, specificity, positive and negative predictive values for this test were evaluated in 102 women with age above 30 years and a palpable breast mass, who were attended at the University of Campinas. All punctures were performed by the same examiner.

Results: the procedure had a sensitivity of $97 \%$, specificity of $87 \%$, positive predictive value of $94 \%$ and negative predictive value of $93 \%$. The insufficient or unsatisfactory sample rate was $16 \%$ for the first aspiration, decreasing to $2 \%$ with a new procedure.

Conclusions: this test showed to be highly sensitive and specific for the differential diagnosis of palpable breast masses, reassuring its great importance for the clinical approach of palpable masses.

KEY WORDS: Fine needle aspiration biopsy. Breast cancer. 


\section{Referências}

1.Alvarenga M. Anatomia patológica e iminuhistoquímica: importância do patologista na mastologia atual. In: Dias EN, Aleffi M, Silva HMS, Figueira Filho ASS. Mastologia atual. Rio de Janeiro: Revinter; 1994. p.127-54.

2.Berg JW, Robbins GF. A late look at the safety of aspiration biopsy. Cancer 1962; 15: 826-7.

3.Butler JA, Vargas HI, Worthen N, Wilson SE. Accuracy of combined clinical-mammographic-cytologic diagnosis of dominant breast masses. A prospective study. Arch Surg 1990; 125: 893-5.

4.Cezar Júnior OP, Magrini E, Brugnerotto K. Punção aspirativa por agulha fina (P.A.A.F.) no diagnóstico dos nódulos mamários. Ginecol Obstet Atual 1997; 1/2: 35-6.

5.Costa MJ, Tadros T, Hilton G, Birdsong G. Breast fine needle aspiration cytology. Utility as a screening tool for clinically palpable lesions. Acta Cytol 1993; 37: 461-71.

6.Fox CH. Inovation in medical diagnosis - the Scandinavian curiosity. Lancet 1979; 1: 1387-8.

7.Freitas Jr R. Penetração de células neoplásicas no trajeto da agulha após PAAF- Relato de caso. J Bras Ginecol 1994; 104: 239-40.

8.Hammond S, Keyhani-Rofagha S, O־Toole RV. Statistical analysis of fine needle aspiration cytology of the breast. A review of 678 cases plus 4,265 from the literature. Acta Cytol 1987; 31: 276-80.

9.Howat AJ, Stringfellow HF, Briggs WA, Nicholson CM. Fine needle aspiration cytology of the breast. A review of 1,868 cases using the Cytospin method. Acta Cytol 1994; 38: 939-44.

10.Kemp C, Valente CA, Terreiro LM, Lima GR. Fine needle aspiration biopsy in diagnosis and treatment of breast pathology. A review. Rev Paul Med 1992; 110: 118-23.

11.Kemp C, Lima GR, Kogos W, Gebrim LH, Alcântara AMP, Miyazawa M. Punção aspirativa por agulha fina: a importância do examinador na obtenção de material para análise - estudo de 261 casos. Rev Bras Ginecol Obstet 1993; 15: 119-22.
12.Koss LG. The palpable breast nodule: a cost-effectiveness analysis of alternate diagnostic approaches. The role of the needle aspiration biopsy. Cancer 1993; 72: 1499-502.

13.Martin HE \& Ellis EB. Biopsy by needle puncture and aspiration. Ann Surg 1930; 92: 169-81.

14.National Cancer Institute. The uniform approach to breast fine-needle aspiration biopsy. Workshop: Fine - Needle Aspiration of Breast. Diagn Cytopathol 1997; 16:295-311.

15.Place R, Velanovich V, Carter P. Fine needle aspiration in the clinical management of mammary masses. Surg Gynecol Obstet 1993; 177: 7-11.

16.Ranieri E, D’Andrea MR, D’Alesio A, Bergomi S, Virno $F$. The integration of diagnostic tests and the role of outpatient surgery in the management of breast disease. Int Surg 1995; 80: 181-4.

17.Reis FJC, Andrade JM, Velludo MASL, Oliveira AS, Feitosa RB, Marana HRC, et al. Punção biópsia aspirativa (PBA) com agulha fina no diagnóstico diferencial de patologias da mama. Rev Bras Ginecol Obstet 1998; 20: 209-13.

18.Robbins GF, Brothers JH III, Eberhart WF, Quan S. Is aspiration biopsy of the breast cancer dangerous to patient? Cancer 1954; 7: 774-8.

19.Rotstein S, Nilsson B, Svane G, Gustavson-Kadaka E. Clinical examination, mammographic findings and cytological diagnosis in patients with breast disorders. Results of 9 years, follow-up. Acta Oncol 1992; 31: 393-7.

20.Sneige N. Fine-needle aspiration of the breast: a review of 1995 cases with emphasis on diagnostic pitfalls. Diagn Cytopathol 1993; 9: 106-12.

21.Stavric GD, Tevcev DT, Kaftandjiev DR, Novak TJ. Aspiration biopsy cytologic method in diagnosis of breast lesions: a critical review of 250 cases. Acta Cytol 1973; 17: 188-90.

22.Zadjela A, Ghossein NA, Pilleron JP, Ennuyer A. The value of aspiration cytology in the diagnosis of breast cancer: experience at the Foundation $\mathrm{Cu}^{-}$ rie. Cancer 1975; 35: 499-506.

23.Willis SL \& Ramzy I. Analysis of false results in a series of 835 fine needle aspirates of breast lesions. Acta Cytol 1995; 39: 858-64. 